\title{
Ultralong photonic nanojet formed by a two-layer dielectric microsphere
}

\author{
Yuecheng Shen, ${ }^{1}$ Lihong V. Wang, ${ }^{2}$ and Jung-Tsung Shen ${ }^{1, *}$ \\ ${ }^{1}$ Department of Electrical and Systems Engineering, Washington University in St. Louis, St. Louis, Missouri 63130, USA \\ ${ }^{2}$ Department of Biomedical Engineering, Washington University in St. Louis, St Louis, Missouri 63130, USA \\ *Corresponding author: jushen@ese.wustl.edu
}

Received May 15, 2014; accepted June 4, 2014;

posted June 9, 2014 (Doc. ID 212170); published July 7, 2014

\begin{abstract}
A photonic nanojet is a highly focused optical beam with a subwavelength waist on the shadow side of the sphere. Successful far-field applications require long nanojets that extend afar. Using the exact Mie theory, we show that ultralong nanojets can be generated using a simple two-layer microsphere structure, using conventional optical materials that are readily available. In particular, we show that for a glass-based two-layer microsphere, the nanojet has an extension of 22 wavelengths. We also show that long nanojets can be formed using semiconductors at infrared frequencies in free space. (c) 2014 Optical Society of America

OCIS codes: (230.3990) Micro-optical devices; (310.4165) Multilayer design; (350.3950) Micro-optics.

http://dx.doi.org/10.1364/OL.39.004120
\end{abstract}

The photonic nanojet phenomenon was first numerically discovered in 2004 [1]. When the incident light is scattered by a homogenous single dielectric sphere, a tightly focused beam with a subwavelength waist emerges on the shadow side of the sphere. The nanojet has potential applications in nanoparticle sensing [2], subwavelength nanopatterning and nanolithography $[\underline{3}, \underline{4}]$, and the realization of a low-loss coupled resonator optical waveguide $[5,6]$. For all applications, it is desired that the nanojets extend as far as possible in the forward direction. Using simple ray optics, it can be shown that the photonic nanojet phenomenon occurs only when the sphere index $n$ is less than 2 [7], which gives an upper bound of $n$ (hereafter, we assume the background is air). It can further be shown that the smaller the index $n$ is, the further the nanojet reaches. Nonetheless, the lowest available index for conventional optical materials at the visible light frequency range is around 1.37 [8]. The nanojet is only several wavelengths long for a microsphere with an index roughly equal to 1.37. Such a shortcoming can be understood by looking at the energy power flow (Poynting vector) around the focal point [Fig. 1(a)]. In such a case, a rapid convergence near the focal point also unavoidably leads to a fast divergence, and thereby a short nanojet. Consequently, to obtain a long nanojet, the key point is to engineer the microsphere so that the power flow near the focal point is essentially parallel so as to have a small angular deviation. Along this direction of reasoning, multilayer microspheres (up to 100 layers) with graded index between $\sqrt{2}$ and 1 have been proposed and shown to exhibit elongated nanojets [9]. Although intricate nanorod metamaterials can achieve such a low index (1.05-1.28 [10]), the fabrication and integration with desired index profile are still challenging. In this Letter, we design a simple two-layer microsphere structure to achieve the required power flow behavior for an enormously long nanojet [Fig. 1(b)], using indices readily available from conventional optical materials. In particular, we show that for a glass-based two-layer microsphere (e.g., $\mathrm{BaF}$ and LaSF), the nanojet has a remarkable extension of 22 wavelengths. Such a capability will make the nanojet more viable in far-field applications.
We start by describing the two-layer microsphere geometry (Fig. 2). The sphere consists of an inner core of radius $R_{c}$ and with a refractive index $n_{c}$, and of a shell with outer radius $R_{s}$ and with an index $n_{s}$. The enabling feature in this design is that the aforementioned power flow behavior can be accomplished if the core index $n_{c}$ is smaller than the shell index $n_{s}\left(n_{c}<n_{s}\right)$. This scenario is different from the previously proposed multilayer microspheres [9] wherein the refractive index monotonically decreases from the core (index $\sqrt{2}$ ) to the outer shell (index 1). For both multilayer and single-microsphere cases, the power flow inside the sphere always converges, while for the proposed two-layer microsphere structure, the power flow inside the sphere experiences alternating convergence and divergence, where the divergence acts to counterbalance and slow down the convergence
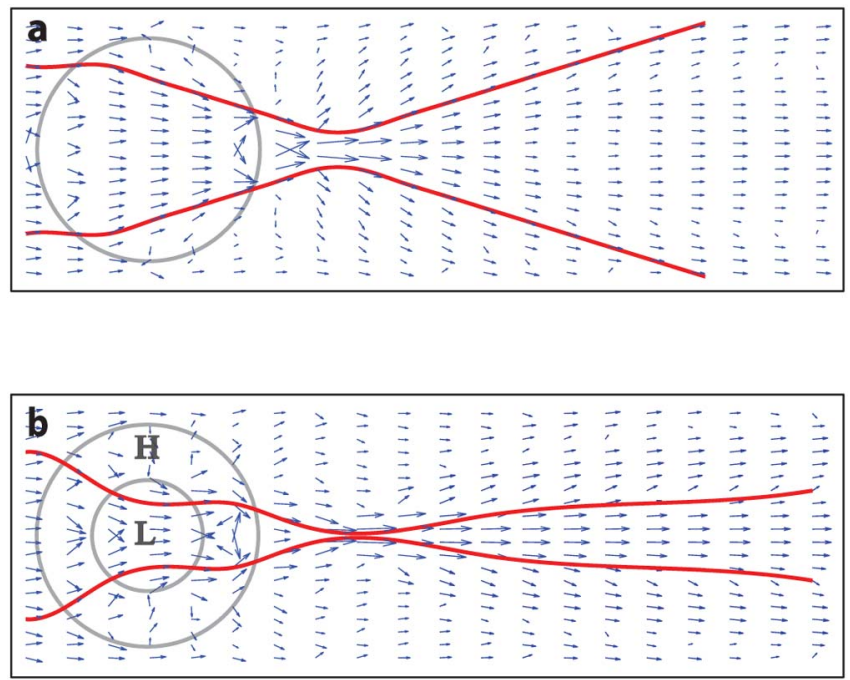

Fig. 1. Power flow plots for microspheres. (a) Single microsphere. (b) Two-layer microsphere. "L" and "H" denote the low index core and high index shell, respectively. The arrows denote the Poynting vector at each grid point. The two continuous red lines in each figure are the streamlines for the Poynting vector fields, assuming the same two fixed starting points on the incident side for each case. 


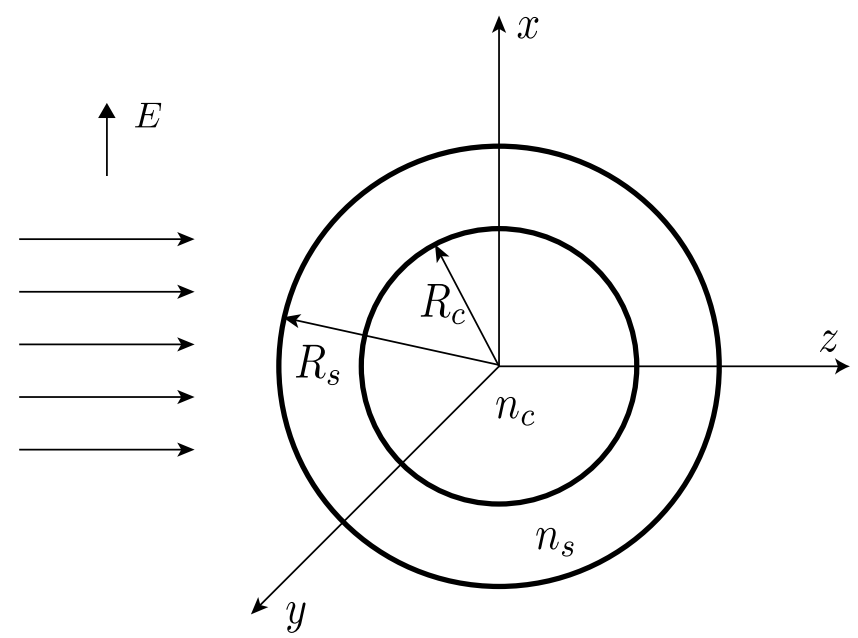

Fig. 2. Schematics of the two-layer microsphere. The sphere consists of an inner core of radius $R_{c}$ and with a refractive index $n_{c}$, and of a shell with outer radius $R_{s}$ and with an index $n_{s}$. The directions of the incident plane wave and the polarization are also denoted.

[Fig. 1(b)]. With properly chosen indices, the counterbalance can be effective so as to minimize the divergence angle at the focal point. We emphasize here that the power flow behavior cannot be correctly inferred from the trajectory of the ray optics, as the dimensions of the layers are of the same orders of magnitude as the wavelength. Fullwave treatment is required to observe numerically correct behavior.

Having introduced the criteria for elongated nanojets, we now plot the intensity distributions of the nanojets from the exact Mie theory [11] to provide a direct visualization of the nanojets in Appendix A. For an incident plane wave propagating along the $z$ direction and linearly polarized in the $x$ direction (Fig. 2), the intensity distributions are shown in Fig. 3. Figure $3($ a) plots the nanojet from a single microsphere, while Fig. $3(\mathrm{~b})$ plots the nanojet from a two-layer microsphere. To plot the intensity distributions, we have chosen the wavelength of the incident light to be $\lambda=632.8 \mathrm{~nm}$ (He-Ne laser), and the radius of the single sphere is $R=5 \lambda, n=1.377\left(\mathrm{MgF}_{2}\right.$ [12]); the twolayer microsphere is designed with $R_{c}=2.5 \bar{\lambda}, n_{c}=1.6028$ (BaF [13]), and $R_{s}=5 \lambda, n_{s}=1.8445$ (LaSF [13]). For different wavelengths, the same geometry also works as long as the indices are kept the same. The intensity profile on the $z$ axis is also plotted for each case. To quantify the extensions of the nanojets, we define the beam length $L$ as the distance on the $z$ axis from the edge of the sphere on the shadow side $(z / \lambda=5$ in the intensity profile plots) to the spatial point where the intensity drops to twice of that of the incident light (the same definition is also adopted in [9]; an alternative practical definition is to compare with the focal peak intensity, which we found does not change the qualitative predictions). The designed twolayer microsphere has a beam length $L \sim 22 \lambda$, which is more than twice as long as that of the single sphere $(L \sim 9 \lambda)$. In Fig. 3 , the intensity profiles in the transverse $y$ direction (beam waist) at the focal point are also plotted in the insets. Although the elongated nanojet in the twolayer microsphere case has a broadened transverse profile compared with that of the single sphere case, its full width at half-maximum (FWHM) is still at subwavelength a
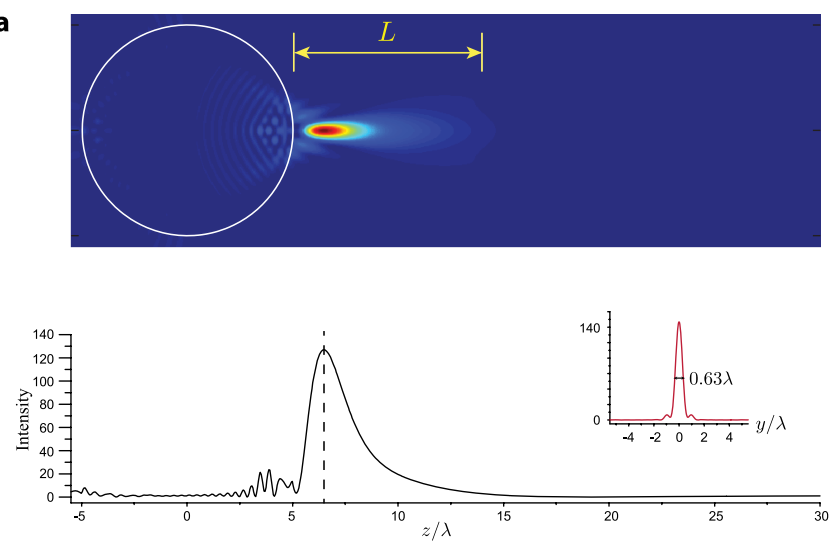

b
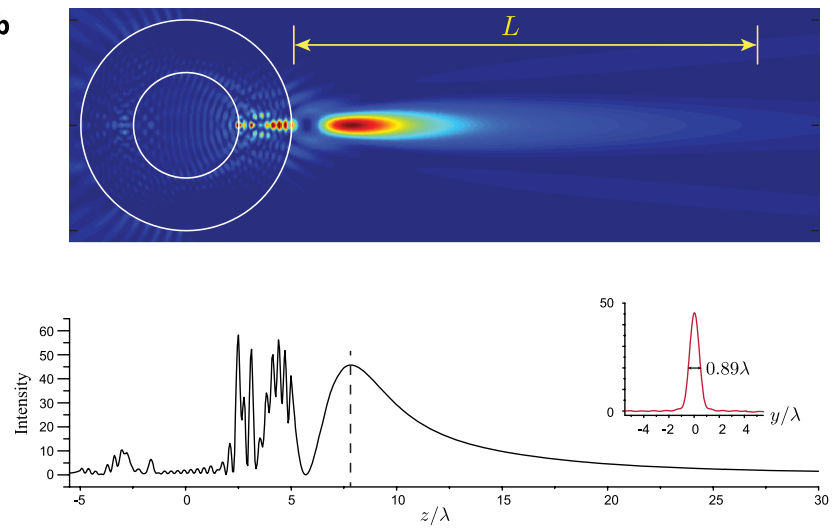

Fig. 3. Intensity plots of the nanojets. (a) Single microsphere with $R=5 \lambda, n=1.377\left(\mathrm{MgF}_{2}\right.$ [12]). (b) A two-layer microsphere with $R_{c}=2.5 \lambda, n_{c}=1.6028$ (BaF [13]), and $R_{s}=5 \lambda$, $n_{s}=1.8445$ (LaSF [13]). In each case, the upper panel plots the intensity for the $y$ - $z$ plane; and the lower panel plots the intensity profile along the $z$ direction. In the intensity profile, the origin of the coordinate system corresponds to the center of the sphere, and the locations of the focal points are denoted by the dashed lines. The transverse profiles at the focal point are also shown in the insets. To clearly show the details of the nanojets, the maximum value of the colormap is chosen to be the value at the focal point. The focal lengths for the two cases are approximately $1.25 \lambda$ and $2.8 \lambda$, respectively.

scale $(\sim 0.89 \lambda)$. We now comment on some properties of the intensity profiles of the nanojets. First, for the twolayer microsphere case, as the nanojet is much longer and slightly wider, energy conservation implies that the intensity at the focal point is accordingly somewhat smaller than that for the single-sphere case (46 versus 127 in units of the intensity of the incoming light). Second, due to the multiple reflections in the outer shell for the two-layer microsphere case, there exist strong standingwave-like interferences in the outer shell. In contrast, the interferences in the single-sphere case are much weaker.

More significantly, using the same low-index core, high-index shell design strategy, we now show that long nanojets can be formed by using materials with index larger than 2 . Such a capability opens up a wide range of materials for nanojet generation. To date, materials with such a large refractive index are excluded from the considerations for nanojet generation for both singlesphere and graded-index multilayer microsphere cases, due to the ray optics predictions and aforementioned 


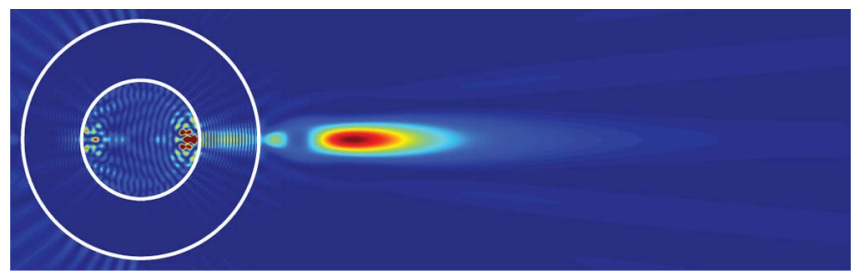

Fig. 4. Long nanojet formed by a two-layer microsphere with high refractive index materials $\left(n_{c}=2, n_{s}=3.85, R_{c}=2.5 \lambda\right.$, and $R_{s}=5 \lambda$ ). Silicon nitride has a refractive index around 2 at the near-infrared frequency range, while many doped semiconductors can have an index range covering 3.85.

monotonically convergent processes. By introducing the counterbalance mechanism for alternating convergence and divergence in the two-layer microsphere structures, our design can accommodate larger index materials $(\geq 2)$ to form long nanojets. Notably, long nanojets in the infrared spectral range based on semiconductor materials (doped and undoped) now become readily feasible. Figure $\underline{4}$ shows an elongated nanojet with $L \sim 15 \lambda$ formed by a two-layer microsphere $\left(R_{c}=2.5 \lambda, n_{c}=2 ; R_{s}=5 \lambda\right.$, $\left.n_{s}=3.85\right)$. The same strong standing-wave-like interferences can also be seen in the outer shell. Here, we note that using semiconductors to form nanojets has been discussed in [1]. In that case, however, the background has an index larger than 1 , so the ratio between the refractive index of the sphere and the background is still less than 2. That is, nanojet generation using semiconductors in air has not been achieved so far. Moreover, we note that the refractive index upper bound $n=2$ given by the ray optics is overestimated. By applying the full-wave analysis, we found that for a single microsphere with $R=5 \lambda$, the largest index to form a nanojet is only around 1.75 , which is $12.5 \%$ smaller than that given by the ray optics.

In conclusion, we designed a simple two-layer microsphere, which can generate an ultralong nanojet, using conventionally available optical materials. We further show that long nanojets can be formed using semiconductor materials at infrared frequencies. This capability will greatly facilitate successful applications of nanojets.

\section{Appendix A}

Here, we provide sufficient mathematical details for the Mie theory used to describe the nanojets in this Letter. For the $x$-polarized incident plane wave with an amplitude $E_{0}$ propagating in the $z$ direction, the electric field $\mathbf{E}$ can be described as linear combinations of the vector spherical harmonics $\mathbf{M}$ and $\mathbf{N}$ (each of which is a function of the spherical coordinates $r, \theta, \phi)[\underline{11}]$ :

$$
\mathbf{E}= \begin{cases}\sum_{n=1}^{\infty}\left(i a_{n} \mathbf{N}_{e 1 n}^{(3)}-b_{n} \mathbf{M}_{o l n}^{(3)}\right) E_{n}, & \text { if } R_{s}<r \\ \sum_{n=1}^{\infty}\left(f_{n} \mathbf{M}_{o l n}^{(1)}-i g_{n} \mathbf{N}_{e 1 n}^{(1)}+v_{n} \mathbf{M}_{o 1 n}^{(2)}-i w_{n} \mathbf{N}_{e 1 n}^{(2)}\right) E_{n}, & \text { if } R_{c}<r<R_{s}, \\ \sum_{n=1}^{\infty}\left(c_{n} \mathbf{M}_{o 1 n}^{(1)}-i d_{n} \mathbf{N}_{e 1 n}^{(1)}\right) E_{n}, & \text { if } r<R_{c}\end{cases}
$$

where $r$ is the radial coordinate and $E_{n}=i^{n} E_{0}(2 n+1) / n(n+1)$. In the vector spherical harmonics, the subscripts $e$ and $o$ denote even and odd modes, respectively; 1 is the azimuthal order, and $n$ is the order of vector spherical harmonics. Each of the superscripts from (A1)-(A3) corresponds to the appropriate choice of one of the four kinds of spherical Bessel functions. By matching the boundary conditions, all the coefficients $\left\{a_{n}, b_{n}, c_{n}, d_{n}, f_{n}, g_{n}, v_{n}, w_{n}\right\}$ can be determined. For example, $a_{n}$ and $b_{n}$ are given by

$$
\begin{aligned}
a_{n} & =\frac{\psi_{n}(y)\left[\psi_{n}^{\prime}\left(m_{2} y\right)-A_{n} \chi_{n}^{\prime}\left(m_{2} y\right)\right]-m_{2} \psi_{n}^{\prime}(y)\left[\psi_{n}\left(m_{2} y\right)-A_{n} \chi_{n}\left(m_{2} y\right)\right]}{\xi_{n}(y)\left[\psi_{n}^{\prime}\left(m_{2} y\right)-A_{n} \chi_{n}^{\prime}\left(m_{2} y\right)\right]-m_{2} \xi_{n}^{\prime}(y)\left[\psi_{n}\left(m_{2} y\right)-A_{n} \chi_{n}\left(m_{2} y\right)\right]} \\
b_{n} & =\frac{m_{2} \psi_{n}(y)\left[\psi_{n}^{\prime}\left(m_{2} y\right)-B_{n} \chi_{n}^{\prime}\left(m_{2} y\right)\right]-\psi_{n}^{\prime}(y)\left[\psi_{n}\left(m_{2} y\right)-B_{n} \chi_{n}\left(m_{2} y\right)\right]}{m_{2} \xi_{n}(y)\left[\psi_{n}^{\prime}\left(m_{2} y\right)-B_{n} \chi_{n}^{\prime}\left(m_{2} y\right)\right]-\xi_{n}^{\prime}(y)\left[\psi_{n}\left(m_{2} y\right)-B_{n} \chi_{n}\left(m_{2} y\right)\right]},
\end{aligned}
$$

where $A_{n}$ and $B_{n}$

$$
\begin{aligned}
& A_{n}=\frac{m_{2} \psi_{n}\left(m_{2} x\right) \psi_{n}^{\prime}\left(m_{1} x\right)-m_{1} \psi_{n}^{\prime}\left(m_{2} x\right) \psi_{n}\left(m_{1} x\right)}{m_{2} \chi_{n}\left(m_{2} x\right) \psi_{n}^{\prime}\left(m_{1} x\right)-m_{1} \chi_{n}^{\prime}\left(m_{2} x\right) \psi_{n}\left(m_{1} x\right)}, \\
& B_{n}=\frac{m_{1} \psi_{n}\left(m_{2} x\right) \psi_{n}^{\prime}\left(m_{1} x\right)-m_{2} \psi_{n}^{\prime}\left(m_{2} x\right) \psi_{n}\left(m_{1} x\right)}{m_{1} \chi_{n}\left(m_{2} x\right) \psi_{n}^{\prime}\left(m_{1} x\right)-m_{2} \chi_{n}^{\prime}\left(m_{2} x\right) \psi_{n}\left(m_{1} x\right)} .
\end{aligned}
$$

Here, $m_{1}$ and $m_{2}$ are ratios of the refractive indices of the core and shell to that of the background medium, respectively. The dimensionless quantities $x$ and $y$ are set to be $k R_{c}$ and $k R_{s}$, respectively. $\psi_{n}(x), \xi_{n}(x)$, and $\chi_{n}(x)$ are the Riccati-Bessel functions. Here, the usage is to keep the notations the same as those used in [11]. Similar expressions for other coefficients can be obtained in the same fashion but will be omitted here. To numerically compute the intensity plots, we sum up the vector spherical harmonics up to order $n=45$. We have checked the convergence such that when order $n=46$ is added, the relative electric field change is less than $10^{-4}$ at all grid points. 


\section{References}

1. Z. Chen, A. Taflove, and V. Backman, Opt. Express 12, 1214 (2004).

2. X. Li, Z. Chen, A. Taflove, and V. Backman, Opt. Express 13, 526 (2005).

3. E. Mcleod and C. B. Arnold, Nat. Nanotechnol. 3, 413 (2008).

4. W. Wu, A. Katsnelson, O. G. Memis, and H. Mohseni, Nanotechnology 18, 485302 (2007).

5. A. Kapitonov and V. Astratov, Opt. Lett. 32, 409 (2007).

6. S. Yang and V. N. Astratov, Appl. Phys. Lett. 92, 26111 (2008).

7. A. Heifetz, S. C. Kong, A. V. Sahakian, A. Taflove, and V. Backman, J. Comput. Theor. Nanosci. 6, 1979 (2009).

8. E. F. Schubert, J. K. Kim, and J. Q. Xi, Phys. Status Solidi B 244, 3002 (2007).
9. S. C. Kong, A. Taflove, and V. Backman, Opt. Express 17, 3722 (2009).

10. L. W. Hrubesh and J. F. Poco, "Method of producing optical quality glass having a selected refractive index," U.S. patent 6,158,244 (12 December 2000).

11. C. F. Bohren and D. R. Huffman, Absorption and Scattering of Light by Small Particles (Wiley, 2008).

12. M. Bass, C. DeCusatis, J. Enoch, V. Lakshminarayanan, G. Li, C. MacDonald, V. Mahajan, and E. V. Stryland, Handbook of Optics, Volume IV: Optical Properties of Materials, Nonlinear Optics, Quantum Optics (McGraw-Hill Professional, 2009).

13. Schottopticalglass datasheet-catalog, http://refractiveindex .info/download/data/2012/schott_optical_glass_collection_ datasheets_dec_2012_us.pdf 